\title{
Cardiac arrhythmias that masquerade as epilepsy
}

\author{
G D SCHOTT, A A MCLEOD, D E JEWITT
}

\section{Results}

Excluding two patients whose ECGs showed minor degrees of axis deviation, which we regarded as irrelevant, 11 patients were considered to have significant underlying arrhythmias, 10 of whom underwent prolonged ambulatory monitoring. One of the patients monitored-a 67-year-old man-was excluded because there was evidence that he had severe cervical spondylosis. Vertebrobasilar arterial disease was thought to contribute significantly to his symptoms, although an ECG showed stable right bundle-branch block and 24-hour ECG monitoring showed a sinus bradycardia during sleep of 49 beats $/ \mathrm{min}$.

No abnormality on the routine ECG was detected in four of the 10 patients with significant arrhythmias. In nine patients the skull and chest radiographs were normal and the EEGs were either normal or showed minor non-specific changes; one patient (case 10) had a transient focal EEG abnormality. The following case reports indicate the variety of presentations with which the 10 patients with suspected epilepsy and significant arrhythmias were referred.

\section{Case reports}

Case 1-This 69-year-old man convulsed for a short period one night and was slightly confused for an hour afterwards. Apart from a little short-lived difficulty in finding his way to work that morning, he remained well, and when seen a few days later he showed no abnormal signs and had a normal ECG. A month later he had two further nocturnal episodes a few days apart. These were associated with cyanosis and convulsions strong enough, on the second occasion, to dislocate his jaw. At examination his pulse was slightly irregular. An ECG recorded shortly afterwards was again normal, but in view of the transient irregular pulse 24-hour ECG monitoring was undertaken. This showed frequent ventricular ectopic beats with salvos of multifocal and unifocal ventricular ectopics (ventricular tachycardia). $\mathrm{He}$ was started on disopyramide and has since remained asymptomatic over six months.

Case 2-A 21-year-old gardener had suffered from birth asphyxia and had had an irregular pulse as a baby. During childhood he had occasional aggressive outbursts, considered to be psychiatric in origin, but an irregular pulse was again noted. In 1974 he developed episodes of unconsciousness lasting up to half an hour, often preceded by chest pain and followed by severe aggressive behaviour for several hours with amnesia for the preceding events. These episodes increased in frequency until they were occurring more than 12 times a month. Tongue biting, incontinence; and convulsions were never observed, though the attacks were often provoked by alcohol. Skull and chest radiographs, routine and sleep EEGs, isotope brain scan, and several fasting blood sugar and calcium determinations were normal. Urine porphyrin screening tests and a serological test for syphilis proved negative. His pulse rate was 40 beats/min, but examination otherwise showed nothing abnormal. A resting ECG showed sinus bradycardia of 50 beats/min and an exercise ECG showed a normal physiological sinus tachycardia at peak exercise, but a $2: 1$ sinoatrial block developed suddenly after this. 24-hour ECG monitoring showed intermittent supraventricular tachycardia, episodes of sinus bradycardia, and frank sinoatrial block, compatible with sinoatrial disease. An endocardial pacing system was inserted, and the symptoms resolved, but a month later periods of unconsciousness returned. The ECG showed that pacing was unsatisfactory and the pacemaker system was replaced with resolution of symptoms once more. A month later the pacemaker failed because the electrode had penetrated the myocardium, and he again suffered episodes of unconsciousness. The pacemaker was again replaced, and the symptoms have not recurred during three months' follow-up.

Case 3-A previously healthy 17-year-old boy suddently lost consciousness while unloading furniture, becoming pale, sweaty, and tremulous. He remained unconscious for 20 minutes but recovered fully after a few minutes. A paternal uncle was said to have had longstanding "epilepsy." Apart from a high resting pulse rate of 100 
and 130 beats/min on two occasions, examination showed nothing abnormal. An ECG rhythm strip recorded at the time of the EEG showed bradycardia that was occasionally less than 45 beats/min, sudden episodes of sinus arrest, and nodal escape beats, consistent with sinoatrial disease. The patient was not investigated further because he repeatedly failed to attend for follow-up.

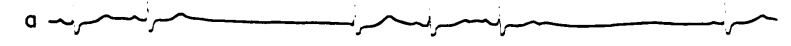

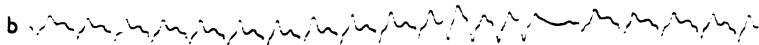

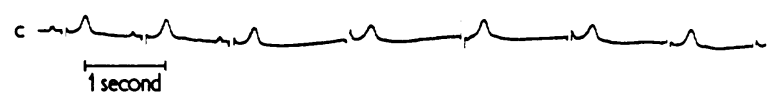

Examples of arrhythmias detected. (a) Case 2 Episodes of sinus arrest. (b) Case 5 Sinus tachycardia at rest and short episodes of supraventricular tachycardia. (c) Case 6 Nodal escape rhythm.

Case 4-A 13-year-old boy, the second twin, was born by a breech delivery and was cyanosed for a short period after birth. For a year he had complained of episodes of muzziness and blankness, particularly on standing, which resolved after a few seconds. Subsequently he had a different form of attack: while reaching up to a shelf he became unconscious and turned round, his eyes became elevated, and he collapsed to the floor for a few seconds, without any colour change, convulsions, tongue biting, or incontinence; after about a minute's confusion he returned to normal. Physical examination showed nothing abnormal. Epilepsy had been diagnosed elsewhere and anticonvulsant treatment started, though without benefit. An ECG and two-minute rhythm strip, however, consistently showed bifid $\mathrm{P}$ waves; several ECGs confirmed sinus rhythm but with rates as low as 48 beats/min. 24-hour ECG monitoring showed sinus bradycardia from 44 beats/min and sinus tachycardia up to 110 beats/min. Varying $\mathrm{P}$-wave configurations ("wandering pacemaker") and second-degree sinoatrial block of Wenckebach type were also seen, although formal electrophysiological studies showed normal intra-atrial and atrioventricular conduction times. The patient suffered increasingly frequent dizzy spells and was reluctant to consider insertion of a pacemaker. Anticonvulsant medication had been withdrawn when he was first seen, and he was started on sustained-release isoprenaline, but this proved ineffective and his treatment remains under review.

Case 5-A 19-year-old man had been born with the umbilical cord around his neck and had probably required resuscitation after birth. He had had faint feelings as a boy, but when he was 18 and while singing on stage during training as a professional singer he suddenly lost consciousness for about 45 minutes and was pale and slightly tremulous but without convulsions, tongue biting, or incontinence. He recovered rapidly; he had a severe headache the next day but otherwise returned to normal. Subsequently he had increasingly frequent episodes of loss of consciousness (up to three a week) each lasting several minutes. On direct questioning he said that he felt chest tightness at the onset of some attacks. He was tall and thin with a high-arched palate. Examination also showed a short mid-systolic basal cardiac murmur and an intermittently irregular pulse. While EEG, skull and chest radiographs, echocardiogram, and urine homocystine excretion were normal, the ECG showed sinus rhythm at $45-50$ beats/min; 24 -hour ECG monitoring showed pronounced sinus bradycardia, atrial tachycardia, periods of supraventricular tachycardia, second-degree sinoatrial block, and considerable variation of sinus rate. After insertion of a demand pacemaker he has had no further syncopal episodes over four months' follow-up, although he has complained of rapid palpitations, which have been effectively treated with metoprolol.

Case 6-This 36-year-old man lost consciousness and became tremulous for about one minute while sitting at a table. The episode was preceded by a few minutes' vertigo but followed by full recovery.
Examination showed nothing abnormal apart from slightly irregular pulse rates of 60 beats $/ \mathrm{min}$ and 76 beats $/ \mathrm{min}$. His father had suffered from collapse attacks but no further details were available. An ECG showed a sinus bradycardia of 46 beats/min ; 24-hour ECG monitoring showed sinus rhythm varying from 35 to 100 beats/min and a junctional escape rhythm of 38 beats $/ \mathrm{min}$. His attacks were probably attributable to bradycardia, but as he had lost consciousness only once we postponed a decision on inserting a pacemaker, and the patient remains under review and without medication.

Case 7-In the past three years this 65-year-old man suffered four episodes of momentary unconsciousness, three while driving and the fourth before micturating. Apart from some mild dyspnoea on exertion and obesity, his health had otherwise been excellent. Examination showed only an irregular pulse of 50 beats $/ \mathrm{min}$; his blood pressure was $100 / 60 \mathrm{~mm} \mathrm{Hg}$ without postural drop. An ECG confirmed sinus bradycardia of $45-50$ beats/min, and 24-hour ECG monitoring showed sinus rhythm varying from 60 to 160 beats $/ \mathrm{min}$, appropriate for his activity, with multifocal ventricular ectopic beats and occasional supraventricular ectopic beats. His attacks of unconsciousness were probably attributable to ventricular arrhythmias, perhaps exacerbated by the stress of driving, and he has been asymptomatic for four months on antiarrhythmic medication with mexilitine.

Case 8-At the age of 18 months this 14-year-old schoolgirl had whooping cough associated with one short episode of generalised convulsions at the height of the fever. When she was 3, and one month after a minor head injury, a second generalised convulsion occurred, possibly in association with breath holding, but examination, skull radiograph, and EEG were unremarkable. From the age of 5 she had had episodes of sweatiness and dizziness, sometimes associated with chest tightness and dyspnoea lasting a quarter of an hour. In view of her history these episodes had been attributed to temporal lobe seizures, but on direct questioning it was apparent that they were often accompanied by rapid, sometimes irregular, heart action. One attack was precipitated by an argument and several by being in crowded places. She never lost consciousness. Examination showed a persistent tachycardia of $100-120$ beats $/ \mathrm{min}$ even when she was relaxed. Catecholamine excretion and thyroid function were normal. The routine ECG was normal. 24-hour ECG monitoring showed second-degree sinoatrial block with sinus tachycardia of up to 160 beats $/ \mathrm{min}$. Since her current symptoms related only to the episodes of tachycardia she was treated with digoxin with reasonable benefit, in an attempt to reduce sinus rate and suppress possible supraventricular rhythms.

Case 9-A 46-year-old photographer suddenly felt hot and nauseated while watching television and shortly afterwards lost consciousness for some minutes. Six weeks later he lost consciousness without warning for a few seconds. Both these episodes resolved without sequelae. Although he was referred with a diagnosis of epilepsy, questioning revealed that he had had three episodes of short-lived rapid heart action in the past year. Examination showed nothing abnormal apart from a pulse rate of 60 beats $/ \mathrm{min}$ on two occasions. While an ECG was normal, 24-hour ECG monitoring showed frequent ventricular ectopic beats, and a couplet of ventricular ectopic beats was also noted. Antiarrhythmic medication with disopyramide was introduced and symptoms have not recurred over the three months he has attended for review.

Case 10-About 12-18 months before referral this 29-year-old doctor had suffered three episodes of clumsiness of the right hand and slight weakness of the right side of the face, each episode diminishing after 10 to 30 minutes and resolving completely after two hours. There was no speech disturbance in this right-handed patient. Examination had shown nothing abnormal. A minimal excess of left frontotemporal theta activity on the EEG was detected on one occasion, but an isotope brain scan was negative. Six months later she sustained the first episode of loss of consciousness. Subsequently, she had eight further episodes each lasting from a few seconds to a few minutes. There were no obvious precipitating factors, and convulsions, tongue biting, and incontinence never occurred. She sometimes recalled having transient giddiness, lightheadedness, and nausea before the episodes and short-lived headaches without confusion afterwards. Immediately after one attack, a tachycardia was noted, but the pulse was not recorded during the attack. Physical examinations have otherwise been unremarkable. Ambulatory ECG monitoring performed elsewhere over a total of 96 hours failed to show significant abnormality, apart from an intermittent slightly prolonged P-R interval up to 0.24 second. She was started on phenytoin but her attacks did not stop. Recent investigations, including computerised tomography of the brain and sleep EEG, 
showed nothing abnormal. In view of recurrent periods of unconsciousness additional prolonged ECG monitoring was undertaken. The first of these further 24-hours of prolonged monitoring showed only sinus arrhythmia; the second, however, showed several episodes of Wenckebach type second-degree atrioventricular block, the overall ventricular response rate often being around 40 beats $/ \mathrm{min}$. Because of the increasingly frequent episodes of unconsciousness, now thought to be due to cardiac arrhythmias, a permanent demand pacemaker was inserted, and the symptoms have disappeared over three months' follow-up.

\section{Discussion}

These case reports emphasise that the effects of cardiac arrhythmias may simulate epilepsy and present to the neurological department more often than is recognised. We have seen several other similar cases, and we have also seen the effects of impaired cerebral perfusion, undoubtedly due to a primary cardiac arrhythmia, closely mimicking the tonic component of a typical epileptic seizure. ${ }^{8}$ Nevertheless, none of the patients we studied suffered symptoms while they were being monitored, so we cannot establish beyond doubt that cardiac arrhythmias were the cause of loss of consciousness. This is especially so since arrhythmias may accompany seizures as a secondary event. ${ }^{9}$

Those patients in whom arrhythmias are most likely to present as epilepsy cannot be clearly identified, though in only one of our ten patients was the EEG significantly (though transiently) abnormal. Focal neurological symptoms, to which emboli arising from the heart may contribute, ${ }^{10}$ do not exclude arrhythmias as a cause. Furthermore, some of our patients suffered prolonged periods of unconsciousness and convulsionsfeatures that are usually considered typical of epilepsy. This again emphasises the link between the heart and the head."

None of our patients had complete atrioventricular heart block, which has been described in many other reports. The reasons for this discrepancy include the fact that many of our patients were young. Arrhythmias are known to be common in elderly people, and most episodes of unconsciousness caused by arrhythmias are reported in this age group; these patients are therefore often referred direct for cardiological assessment. ${ }^{1}$ Neither age nor duration of symptoms excludes arrhythmias as a cause of epilepsy, and we suspect that among the wide variety of arrhythmias known to be capable of causing loss of consciousness, previously asymptomatic sinoatrial disease may be particularly common..$^{12} 13$

Arrhythmias undoubtedly underlie epilepsy more often than conditions such as hypoglycaemia and hypocalcaemia that are more frequently considered, but from our study we cannot estimate their frequency precisely. The number of patients studied was small; disorders among patients attending a neurological department may not reflect their frequency in the general population; the initial diagnosis with which a patient is referred is often arbitrary or imprecise; and patients with a wider range of symptoms, including those with typical, established and perhaps anticonvulsant-responsive epilepsy, might be found to have significant underlying arrhythmias after appropriate investigation. Nevertheless, we have shown relevant arrhythmias in about $20 \%$ of the patients with suspected idiopathic epilepsy who were seen over six months. Even if patterns of referral differ widely, the number of affected patients seems to be remarkably high, although it is consistent with the frequency with which syncope and dizziness can be correlated with cardiac arrhythmias, provided sufficiently prolonged ECG monitoring is undertaken. ${ }^{14}$

Any attempt to assess the frequency with which arrhythmias contribute to epileptic symptoms must take into account several other factors. Cardiac and cerebral disease may coexist, particularly in the elderly (see case 1). Moreover, patients with underlying cerebral damage may be more susceptible to insufficiency of the cerebral circulation: four of our patients, all of whom presented at a relatively young age, had a history of early childhood convulsions or misadventure at birth, during which time cerebral damage may have occurred. This emphasises the fact that in some patients cerebral disorders can be clearly assessed only after cardiac factors have been excluded. It is also of interest that death from myocardial infarction appears to be uncommon among epileptic patients $;^{15}$ we do not know the extent to which anticonvulsants with antiarrhythmic properties, such as phenytoin, have proved effective in "epileptic" patients with underlying cardiac arrhythmias. A family history of epilepsy also cannot exclude underlying arrhythmias, the familial occurrence of arrhythmias presenting with loss of consciousness being well recognised. ${ }^{16}$

Routine ECGs are rarely recorded in the investigation of epilepsy. We consider, however, that the finding of unexplained tachycardia, irregular pulse (when physiological sinus arrhythmia has been excluded), and especially bradycardia ( $<50$ beats/ min)-which was present in four of our patients-demands further investigation. This is particularly so when the typical clinical features of epilepsy are absent and the EEG shows nothing abnormal. Only one of our patients had other cardiac abnormalities and the diagnosis has depended on the detection of cardiac rhythm changes only.

A standard ECG in a patient suffering from periods of unconsciousness will often be normal, and, as our study has confirmed, continuous ECG monitoring of an ambulant patient may be necessary to show abnormalities. ${ }^{414}$ Even so, one of our patients (case 10) underwent over 120 hours' monitoring before a serious arrhythmia was detected. The role of prolonged monitoring, particularly when the suspicion of relevant arrhythmia is slight, will have to be carefully assessed. Indeed, the "normal" heart rhythm will have to be reconsidered and defined, since a remarkably high incidence of arrhythmias has been detected in asymptomatic subjects after prolonged ECG monitoring. ${ }^{17}$ Nevertheless, as awareness of these conditions has grown, increasing numbers of patients (currently three or four a month) have been referred for cardiological assessment and prolonged monitoring, and there has been a corresponding increase in the numbers of significant arrhythmias detected.

The indications for treating these arrhythmias also require consideration. A single episode of loss of consciousness associated with complete atrioventricular heart block would clearly have to be treated, but it is not so clear whether a single episode of loss of consciousness, with which a new "epileptic" patient is commonly referred to a neurological department, and attributable, for instance, to sinoatrial disease, should be treated. Most neurologists hesitate to recommend prolonged anticonvulsant medication after a single fit in an otherwise healthy person.

As a result of our observations we consider that cardiac arrhythmias cause or contribute to "idiopathic epilepsy" surprisingly often. We have been concerned with the problems of early management of patients' symptoms; and the indications for performing a routine ECG and prolonged ambulatory ECG monitoring require further investigation, as does the long-term follow-up of patients in whom arrhythmias have been detected and treated. We hope that the increasing recognition and treatment of patients with unsuspected cardiac arrhythmias who present to a neurological department may reduce the number of "epileptic" patients whose disorder remains idiopathic.

We are grateful to the consultant physicians, King's College Hospital, and Professor P K Thomas, The Royal Free Hospital, for permission to report details of patients under their care. We are indebted to Dr K J Zilkha and Professor C D Marsden for their valuable comments and discussion, and thank Miss $F$ Weaver for technical help with the 24-hour electrocardiographic monitoring.

Requests for reprints should be addressed to Dr A A McLeod, Department of Cardiology, King's College Hospital, Denmark Hill, London SE5 9RS. 


\section{References}

${ }^{1}$ McAllen, P M, and Marshall, J, Lancet, 1973, 1, 1212.

2 Abdon, N-J, and Malmcrona, R, Acta Medica Scandinavica, 1975, 198 455.

${ }^{3}$ Walter, P F, Reid, S D, and Wenger, N K, Annals of Internal Medicine $1970,72,471$.

4 Goldberg, A D, Raftery, E B, and Cashman, P M M, British Medical fournal, 1975, 4, 569.

'Sharpey-Schafer, E P, British Medical fournal, 1956, 1, 506.

${ }^{6}$ Hirsch, C S, and Martin, D L, Neurology (Minneapolis), 1971, 21, 682.

${ }^{7}$ Krasnow, A Z, and Bloomfield, D K, American Heart fournal, 1976, 91, 349.

* Driver, M V, and Selby, P J, Electroencephalography and Clinical Neurophysiology. In press.
${ }^{9}$ White, P T, et al, Neurology (Minneapolis), 1961, 11, 354.

${ }^{10}$ Rubenstein, J J, et al, Circulation, 1972, 46, 5

11 British Medical fournal, 1976, 4, 1158.

12 Easley, R M, and Goldstein, S, American fournal of Medicine, 1971, 50, 166.

${ }^{13}$ Fairfax, A J, and Lambert, C D, fournal of Neurology, Neurosurgery, and Psychiatry, 1976, 39, 576.

14 Van Durme, J P, American Heart fournal, 1975, 89, 538.

15 Lindén, V, British Medical fournal, 1975, 2, 87.

16 Saracheck, N S, and Leonard, J J, American fournal of Cardiology, 1972, 29, 451 .

17 Clarke, J, et al, Lancet, 1976, 2, 508.

(Accepted 25 March 1977)

\title{
Today's Treatment
}

\section{Diseases of the urinary system}

\section{Treatment of glomerulonephritis by drugs}

\author{
J S CAMERON
}

British Medical fournal, 1977, 1, 1457-1459

The story of attempts to treat glomerulonephritis is one of hopes raised, later to be dashed-despite the fact that we now possess some understanding of what processes underly the major forms of glomerulonephritis. The advances that we have made have been the result of fortunate accident, rather than inspired logic.

The major advances in managing patients suffering from glomerulonephritis have been the relief of oedema by powerful diuretics, the treatment of infection by antibiotics, and the control of hypertension by hypotensive drugs. These treatments are available to any patient, and the fact that they do not influence the underlying disease should not let us underestimate them; particularly since it seems almost certain that control of hypertension can prolong life and preserve renal function.

This happy story is not, however, the subject of this essay. Here, we must attempt the more depressing exercise of judging our ability to manipulate the events leading to glomerular injury in glomerulonephritis. It must be said straight away that many patients with different forms of glomerulonephritis do not need any specific treatment, even though they may be quite ill. An example is classic post-streptococcal glomerulonephritis with endocapillary proliferation within the glomeruli, but without extracapillary crescent formation. Even though severe hypertension may be seen in the acute phase, or oliguria and uraemia even need dialysis, there is no evidence that treatment of the nephritis is needed, or that full recovery cannot take place. Similarly, children and young adults who suffer repeated attacks of haematuria, and show IgA in the mesangium of their glomeruli with normal histological pattern on optical microscopy, rarely go into uraemia. One-quarter of adult

Guy's Hospital Medical School, London SE1 9RT

J S CAMERON, MD, FRCP, professor of renal medicine patients with membranous glomerulopathy, and at least half the children with a similar appearance, will show complete clinical resolution of the disease within two years; treatment can only harm them with its unwanted side effects. Thus, treatment in glomerulonephritis, especially when its effect is uncertain, should be reserved for groups of patients identified histologically and clinically as those most likely to do badly, and least likely to show spontaneous remission.

We know that most forms of glomerulonephritis appear to arise from the formation, circulation, and glomerular deposition of immune complexes, which can induce an inflammatory response. These events, and the resultant glomerular scarring, lead to the proteinuria, haematuria, hypertension, and loss of filtering surface that characterise glomerulonephritis clinically. The various histological appearances represent the glomerular reaction to various rates of deposition of complexes of different composition, size, and inflammatory potential. The fundamental lesion in the nephritic individual is a relative inefficiency in eliminating foreign antigens, which persist in the circulation in the presence of the antibody response, and may thus form large amounts of soluble complexes. This view of glomerulonephritis suggests that it is the result of various forms of relative immune incompetence-that is, a manifestation of immunodeficiency. A little glomerular disease results from the combination of antibody with specific antigens on the glomerulifor instance, antiglomerular basement membrane antibody nephritis.

\section{Antigen removal}

The various therapeutic attacks on this condition are summarised in table I. Although we have identified over 30 different antigens in occasional cases of glomerulonephritis, in most patients the antigen cannot be removed as it is unknown. Many of the antigens in human glomerulonephritis may, in fact, be the common pathogens to which we are all susceptible and the major lesion, mentioned above, may lie with the host. We can, however, remove the antigen in some patients because it includes the treatment of a persistent infection. The treatment of 